Case Report

\title{
A Case Report of Nonvasculitic Autoimmune Inflammatory Meningoencephalitis with Sensory Ganglionopathy: A Rare Presentation of Sjögren Syndrome
}

\author{
João Peres, ${ }^{1}$ Simão Cruz, ${ }^{1}$ Rita Oliveira, ${ }^{2}$ Luís Santos, ${ }^{3}$ and Ana Valverde ${ }^{1}$ \\ ${ }^{1}$ Neurology Department, Hospital Professor Doutor Fernando Fonseca, Amadora, Portugal \\ ${ }^{2}$ Anatomic Pathology Department, Hospital Professor Doutor Fernando Fonseca, Amadora, Portugal \\ ${ }^{3}$ Neurology Department, Hospital de Egas Moniz-Centro Hospitalar Lisboa Oeste, Lisbon, Portugal \\ Correspondence should be addressed to João Peres; jpperes13@gmail.com
}

Received 30 August 2016; Accepted 12 December 2016; Published 15 January 2017

Academic Editor: Ritsuo Hashimoto

Copyright (C) 2017 João Peres et al. This is an open access article distributed under the Creative Commons Attribution License, which permits unrestricted use, distribution, and reproduction in any medium, provided the original work is properly cited.

\begin{abstract}
A 68-year-old Caucasian female was admitted to the emergency department with a progressive history of behavioural symptoms and anxiety followed by visual and auditory hallucinations, forgetfulness, and impaired gait in the previous 3 months. On examination she was psychotic and had a postural and rest tremor of the upper limbs, cogwheel rigidity of the four limbs, retropulsion on standing position, and inability to walk. During the following 2 weeks she developed xerostomia and unilateral parotiditis that improved with steroids. A simultaneous improvement of the cognitive abilities allowed for the detection of sensory ataxia of the lower limbs. Sensory ganglionopathy was then detected with electrophysiological studies. A diagnosis of Sjögren syndrome was suspected and confirmed by salivary gland scintigraphy, Schirmer's test, and submaxillary gland biopsy. We report a case of Sjögren syndrome associated with central and peripheral nervous system involvement, without sicca symptoms preceding the neurological clinical picture. The coexistence of ganglionopathy and a favourable response to immunosuppression are key features that can lead to the correct diagnosis in cases with atypical CNS symptoms, mimicking a rapidly progressive dementia.
\end{abstract}

\section{Introduction}

Sjögren syndrome is a common autoimmune disease that can be either primary or secondary to other autoimmune diseases. Its diagnosis is often difficult because of the wide clinical heterogeneity. Its histopathologic hallmark is the periductal lymphocytic infiltrate with destruction of salivary and lacrimal glands resulting in loss of their function, which leads to sicca symptoms. Parallel mononuclear infiltrate or vasculitic lesions may affect other organs and systems. Nervous system is one of the most common extraglandular involved sites, and it is reported to precede the sicca symptoms in a proportion of patients [1]. Neurological manifestations can involve both peripheral and central nervous systems. In this case the authors present a case of Sjögren syndrome which manifested as rapidly progressive dementia with impaired gait, in the absence of previous sicca symptoms.

\section{Case Report}

A 68-year-old Caucasian woman was taken by her son to the emergency department with a 3-month progressive history of anxiety followed by complex visual and auditory hallucinations, forgetfulness, and impaired gait in the previous 2 weeks. She had a history of Hashimoto thyroiditis for over 10 years and was on thyroid hormone replacement therapy with levothyroxine, without other relevant personal or family history. General physical examination was unremarkable. On admission she was alert, agitated, with frightened appearance and looking around with suspiciousness. She showed space and time disorientation, impaired attention, and incoherent speech, verbalizing nonsystematized delusional thoughts of persecutory type. Cranial nerve examination was normal. She had a postural and rest upper limbs tremor, cogwheel rigidity of the four limbs, brisk deep tendon reflexes, and a tendency 


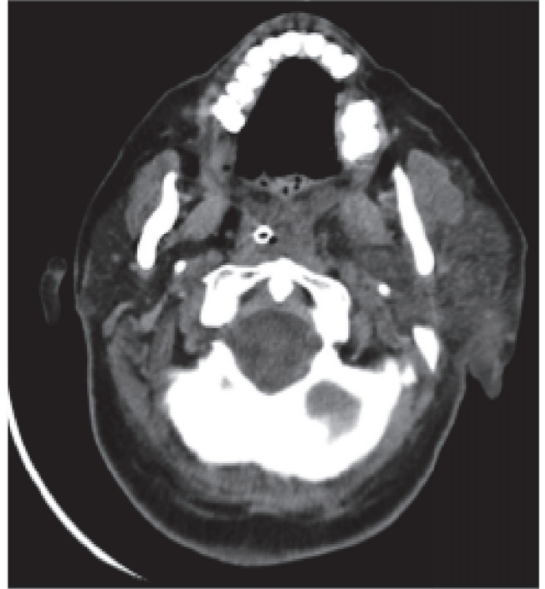

FIGURE 1: Head CT demonstrating left parotiditis.

to fall backwards on standing position and was unable to walk.

Diagnostic workup was performed with an initial clinical hypothesis of a rapidly progressive dementia. CSF examination revealed no cells and an increased protein content ( $74 \mathrm{mg} / \mathrm{dL}$ of the IgG type) with absent oligoclonal bands and with normal serum immunoglobulin concentration. Test for 14-3-3 protein was negative. EEG detected a generalized slowing of background activity, without epileptiform activity. Brain MRI showed only mild diffuse cortical atrophy.

On day 7 after admission, the patient developed xerostomia and unilateral left parotiditis (Figure 1) that remitted under oral prednisolone $40 \mathrm{mg}$ once a day. This treatment also made a marked improvement in cognitive and psychiatric symptoms over the following days. Neurological reassessment on the 14th day showed weak deep tendon reflexes and decreased vibration and position senses on lower limbs, with sensory ataxia, Romberg sign, and wide-based gait. Nerve conduction studies revealed absent sensory nerve action potentials of sural nerve bilaterally, with slight decrease of the amplitude of the compound motor action potentials in the common peroneal and tibial nerves. Needle EMG was unremarkable. Somatosensory evoked potentials were compatible with a dysfunction of the posterior cord below cervical level. The clinical setting and electrophysiological studies were compatible with the diagnosis of a sensory ganglionopathy.

With this clinical scenario and steroid responsiveness, an immune-mediated disease was suspected. The complaints of xerostomia along with the parotiditis were suggestive of Sjögren syndrome. Anti-Ro (SSA) and anti-La (SSB) antibodies were repeatedly negative, as were rheumatoid factor and antinuclear antibodies (ANA). A salivary gland scintigraphy (99TcO4-) was performed and it revealed severely decreased uptake in submaxillary and parotid glands (Figure 2). Schirmer's test showed severe reduction of tear production $(2 \mathrm{~mm}$ bilaterally after 5 minutes) and submaxillary gland biopsy (Figure 3) identified a multifocal inflammatory lymphocytic infiltrate with $>1$ focus $/ 4 \mathrm{~mm}^{2}$ ( 1 focus $=50$ lymphocytes), thus favouring the diagnosis of Sjögren syndrome.

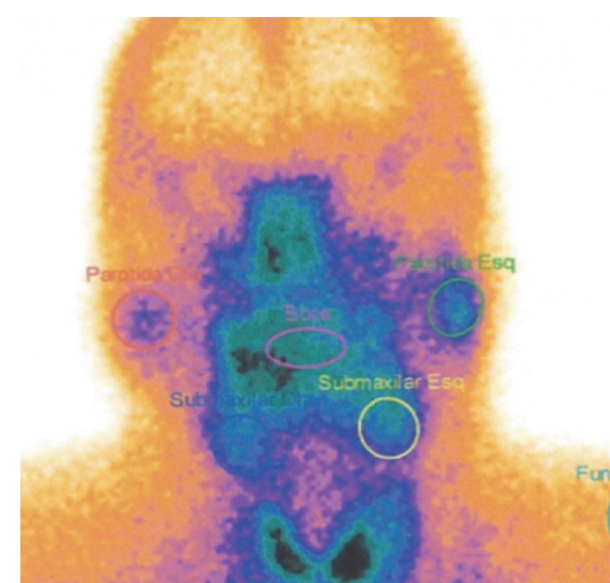

FIGURE 2: Salivary gland scintigraphy showing decreased uptake in submaxillary and parotid.

Steroid therapy resulted in global improvement of psychiatric and cognitive symptoms, as well as of extrapyramidal features and sensory ataxia allowing for unassisted gait. Two months after admission there was a relapse of psychotic symptoms (delusions - the patient strongly believed that her son was in great danger; visual and tactile hallucinations-she saw bizarre animals and felt them touching her legs) that were refractory to oral steroids and antipsychotic drugs, which led to treatment escalation with cyclophosphamide. After 2 cycles of cyclophosphamide completed, recurrent urinary tract infections forced its withdrawal, with subsequent worsening of central and peripheral neurologic symptoms. Rituximab treatment was then initiated, in a regimen of two $1000 \mathrm{mg}$ IV repeated twice with six months apart. Following this treatment, a progressive clinical improvement was observed.

One year after admittance, neurological reassessment showed that the patient was oriented in time and space, with attention and short- and long-term memory preserved. Cranial nerve examination was unremarkable. The superior limbs showed a dystonic posture of the wrist with athetosis of the fingers that were enhanced with the eyes closed.

Normal Strength and Muscle Tone. Absent deep tendon reflexes, decreased vibration, and position senses on the four limbs, with sensory ataxia and Romberg sign, were observed. Gait was wide-based but possible with little assistance. The patient had no psychiatric symptoms and was independent in all basic daily life activities (Figure 4).

\section{Discussion}

In primary Sjögren syndrome, neurological manifestations can be protean and involve both peripheral and central nervous systems, as exemplified by our patient, whose clinical picture included cognitive impairment, hallucinations, extrapyramidal features, and sensory ataxia. To the best of our knowledge, such severe, simultaneous, and rapidly progressive symptoms have not been previously reported. 


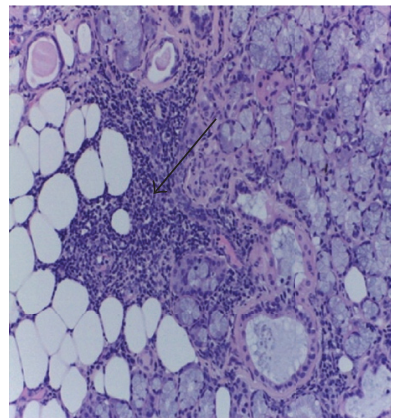

(a)

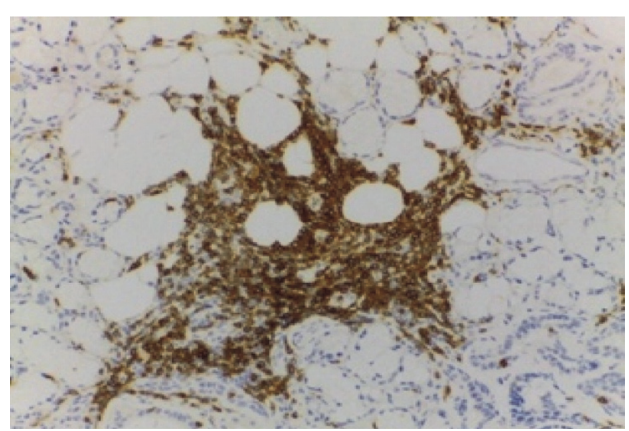

(b)

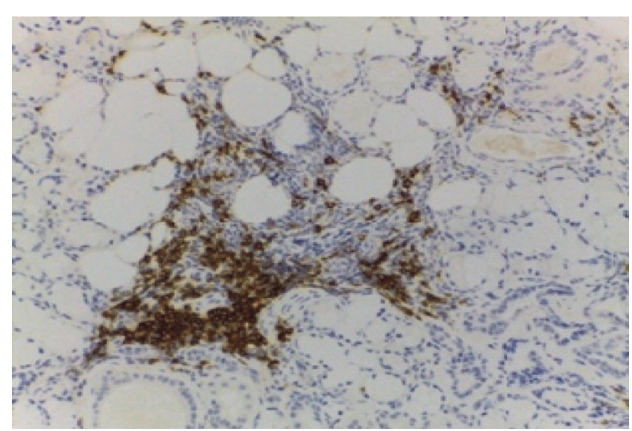

(c)

Figure 3: Submaxillary gland biopsy. (a) H\&E $10 \times 20$-one focus of lymphocytic infiltrate (black arrow). (b) Immunohistochemistry-T lymphocytes (CD3+). (c) Immunohistochemistry-B lymphocytes (CD20+).

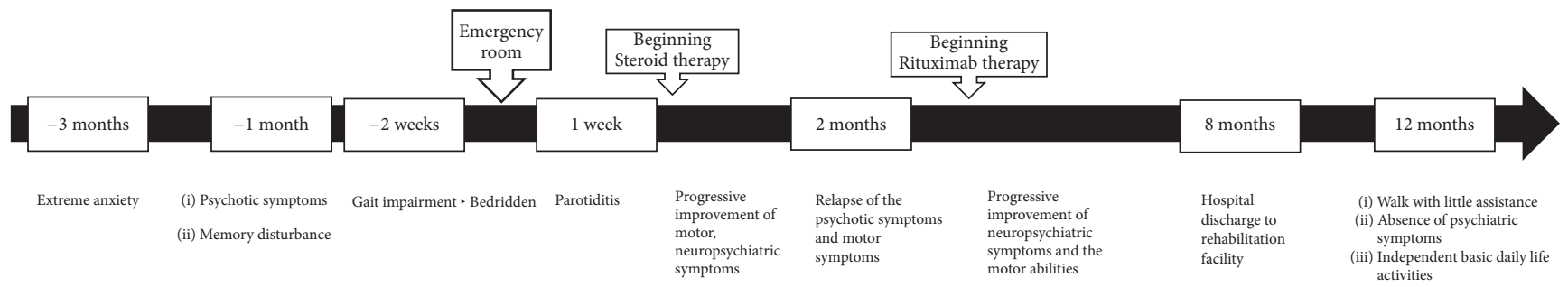

FiguRE 4: Diagram representing the timeline of the relevant clinical events. The time on the arrow refers to the date of emergency room admittance.

The exact pathophysiology of CNS clinical features remains unclear. It is thought to be related to an inflammatory process, affecting small vessels, without causing visible structural damage [2]. Some authors suggest other mechanisms such as direct infiltration of the CNS by mononuclear cells [1]. The term Nonvasculitic Autoimmune Meningoencephalitis was proposed by Caselli et al. in 1999 when describing 5 patients presenting with progressive cognitive decline, with both serologic evidence of autoimmunity and of tissue inflammation, benefiting from immunosuppression. Interestingly, two out of the five patients from this original report later received the diagnosis of Sjögren syndrome [3].

There is no clear topographical relationship between the clinical features and the imaging studies; hence brain MRI is not useful for diagnosis, showing abnormalities in only $30 \%$ of cases [4]. In this particular case no functional imaging was performed, so no clinical-anatomical correlation was done.

Regarding the peripheral nervous system involvement, there is no certainty about the mechanism either. Sensory ganglionopathy appears to be mediated by direct mononuclear infiltration and destruction of the dorsal root ganglion and the posterior columns of the spinal cord, promoted by the leaky basement membrane of the capillaries supplying these structures [1].

Since 1965 several sets of criteria were proposed for classification of Sjögren syndrome. The most widely accepted are the American-European Consensus Group (AECG) criteria (from 1996, later revised in 2002), with no significant sensitivity and specificity difference to the criteria recently issued by the American college of rheumatology (2012) [5]. Our patient meets Sjögren syndrome criteria according to AECG. SS-A and SS-B antibodies were negative, but their low sensitivity (40\%) when nervous system is involved has been previously reported [4].

There is scarce evidence in the literature about Sjögren syndrome treatment. Data regarding efficacy of corticosteroids and other immunosuppressive agents in extraglandular involvement derive from studies designed for sicca symptoms. There is some evidence that Rituximab can be used as a rescue therapy in refractory extraglandular features [6].

\section{Conclusions}

(i) Systemic autoimmune disturbances can affect both central and peripheral nervous system and they can present subacutely.

(ii) SS-A and SS-B antibodies are less sensitive in Sjögren syndrome when nervous system is involved.

(iii) When neurological features occur in Sjögren syndrome they frequently precede sicca symptoms.

(iv) Before the clinical picture of a rapidly progressive cognitive decline, a systemic and treatable cause must be ruled out. Once infectious cause is excluded, immunosuppression should be attempted, and a positive response should motivate an obstinate search for a specific immunomediated disease [7].

\section{Competing Interests}

The authors declare that there is no conflict of interests regarding the publication of this paper. 


\section{References}

[1] G. J. Tobón, J.-O. Pers, V. Devauchelle-Pensec, and P. Youinou, "Neurological disorders in primary sjögren's syndrome," Autoimmune Diseases, vol. 2012, Article ID 645967, 11 pages, 2012.

[2] F. C. Soliotis, C. P. Mavragani, and H. M. Moutsopoulos, "Central nervous system involvement in Sjögren's syndrome," Annals of the Rheumatic Diseases, vol. 63, no. 6, pp. 616-620, 2004.

[3] R. J. Caselli, B. F. Boeve, B. W. Scheithauer, J. D. O’Duffy, and G. G. Hunder, "Nonvasculitic autoimmune inflammatory meningoencephalitis (NAIM): a reversible form of encephalopathy," Neurology, vol. 53, no. 7, pp. 1579-1581, 1999.

[4] S. Delalande, J. de Seze, A.-L. Fauchais et al., "Neurologic manifestations in primary Sjögren syndrome: a study of 82 patients," Medicine, vol. 83, no. 5, pp. 280-291, 2004.

[5] A. Rasmussen, J. A. Ice, H. Li et al., "Comparison of the American-European Consensus Group Sjögren’s syndrome classification criteria to newly proposed American College of Rheumatology criteria in a large, carefully characterised sicca cohort," Annals of the Rheumatic Diseases, vol. 73, no. 1, pp. 31-38, 2014.

[6] M. Ramos-Casals, R. Solans, J. Rosas et al., "Primary Sjögren syndrome in Spain: clinical and immunologic expression in 1010 patients," Medicine, vol. 87, no. 4, pp. 210-219, 2008.

[7] G. S. Day and D. F. Tang-Wai, "When dementia progresses quickly: a practical approach to the diagnosis and management of rapidly progressive dementia," Neurodegenerative disease management, vol. 4, no. 1, pp. 41-56, 2014. 


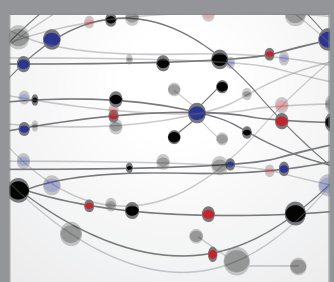

The Scientific World Journal
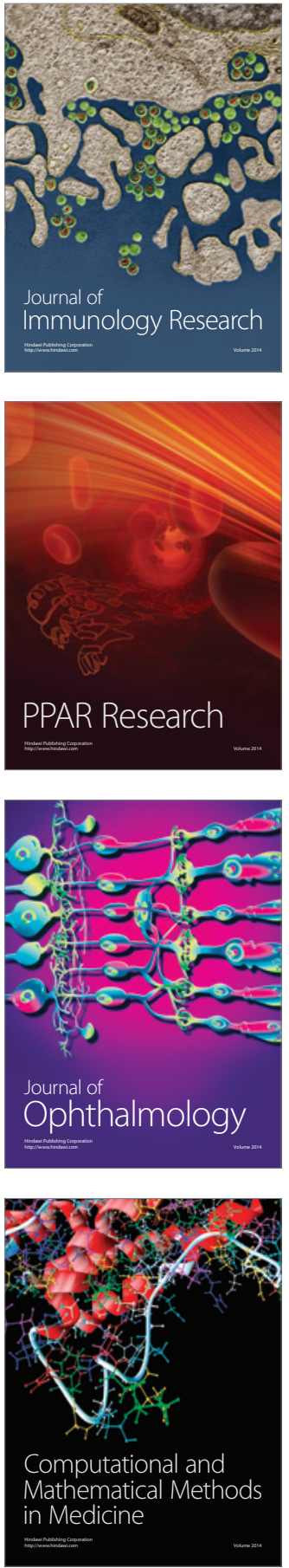

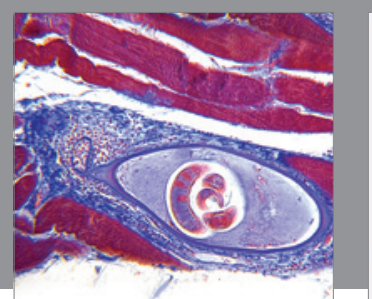

Gastroenterology Research and Practice
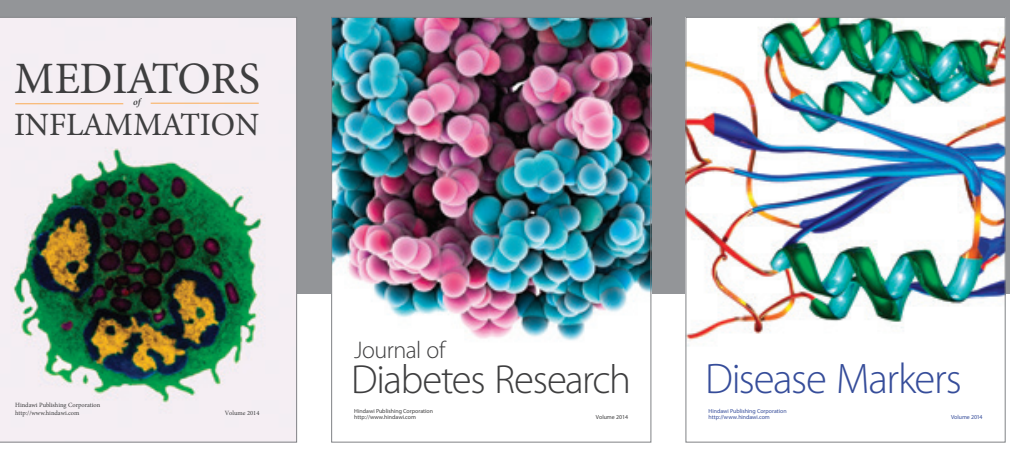

Disease Markers

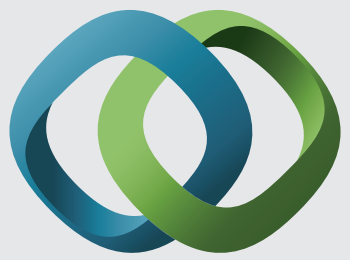

\section{Hindawi}

Submit your manuscripts at

https://www.hindawi.com
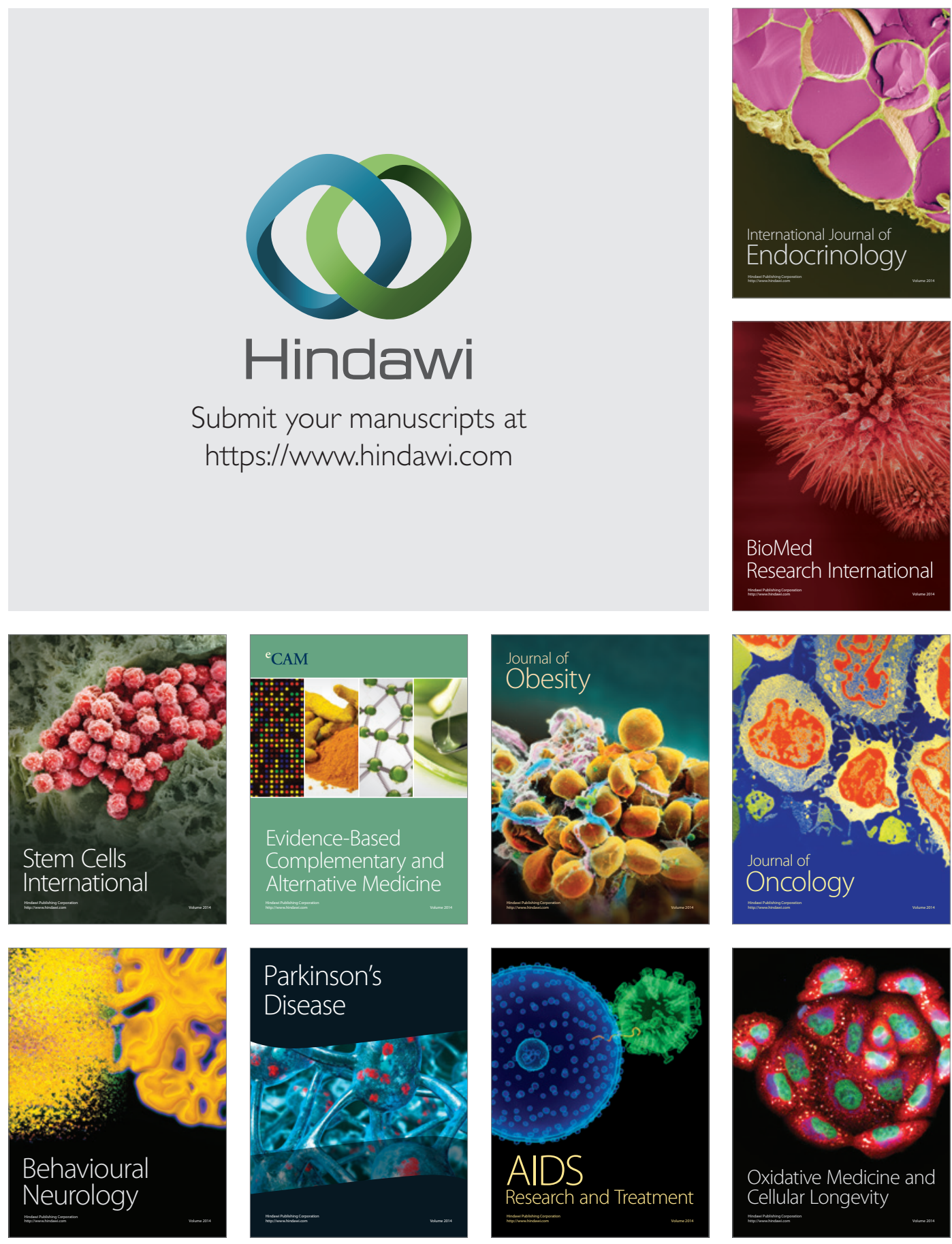